Supporting Information for

\title{
Diamidonaphthalene-Stabilized N-Heterocyclic
}

\section{Pnictogenium Cations and Their Cation-Cation Solid}

\section{State Interactions}

Heather A. Spinney, ${ }^{1}$ Ilia Korobkov, ${ }^{1}$ Gino A. DiLabio, ${ }^{2}$ Glen P. A. Yap, ${ }^{3}$ and

\author{
Darrin S. Richeson ${ }^{1}$
}

${ }^{1}$ Center for Catalysis Research and Innovation, Department of Chemistry, University of Ottawa, Ottawa, Ontario, Canada, K1N 6N5; National Institute for Nanotechnology, National Research Council of Canada, 11421 Saskatchewan Drive, Edmonton, Alberta, Canada T6G 2M9; ${ }^{3}$ Department of Chemistry and Biochemistry, University of Delaware, Newark, Delaware, 19716.

\begin{tabular}{|l|r|}
\hline $\begin{array}{l}\text { 1. The LUMO and pnictogenium lone pair } \\
\text { orbitals for 10a, b, and } \mathbf{d}\end{array}$ & 3 pages \\
\hline $\begin{array}{l}\text { 2. Results of HOMO-LUMO transition } \\
\text { calculations for the } \pi \text {-stacked } \\
\text { pnictogenium complexes }\end{array}$ & 2 pages \\
\hline 3. Full citation for reference 19 & 1 page \\
\hline $\begin{array}{l}\text { 4. Thermal ellipsoid plots for compounds } \\
\text { 9b and 11b. }\end{array}$ & 2 pages \\
\hline $\begin{array}{l}\text { 5MR data for N,N'-diphenyl-1,8- } \\
\text { diaminonaphthalene. }\end{array}$ & 1 page \\
\hline
\end{tabular}



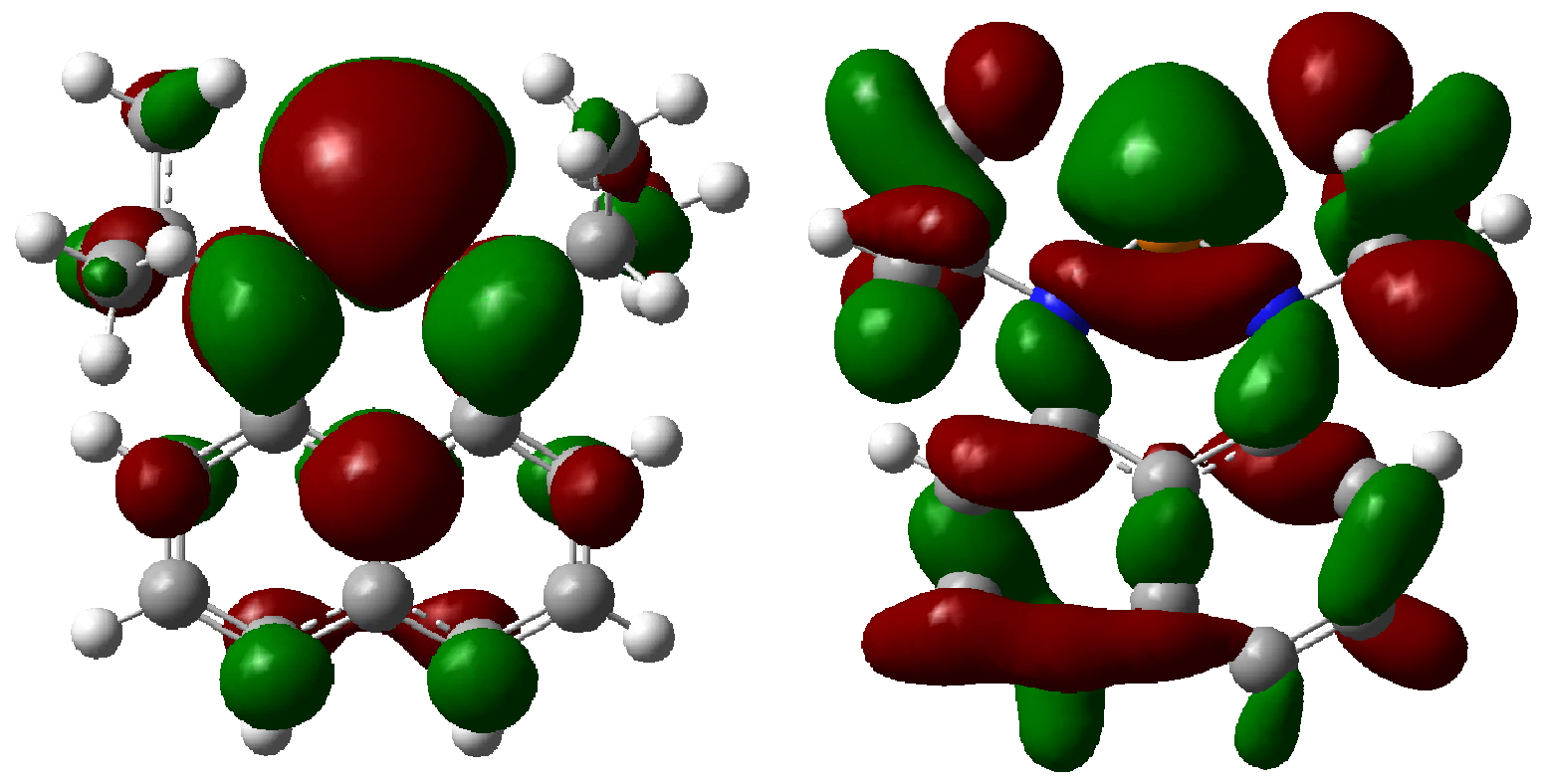

Figure S1. Orbital contour plots of 10a showing the LUMO (left) and P-centered lone pair HOMO-4 (right) obtained from DFT calculations. 

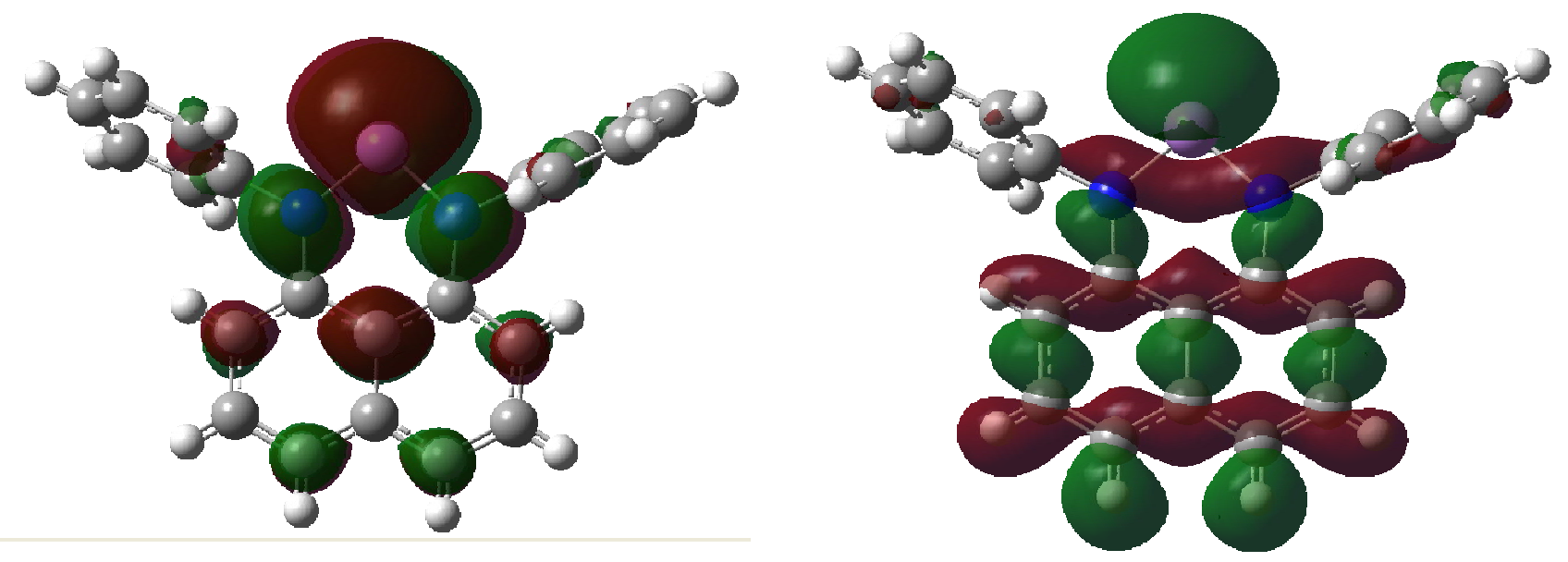

Figure S2. Orbital contour plots of $\left[\mathrm{As}(\mathrm{PhN})_{2} \mathrm{C}_{10} \mathrm{H}_{6}\right] \mathrm{GaCl}_{4}$ 10d. showing the LUMO (left) and As-centered lone pair HOMO-9 (right) obtained from DFT calculations. 

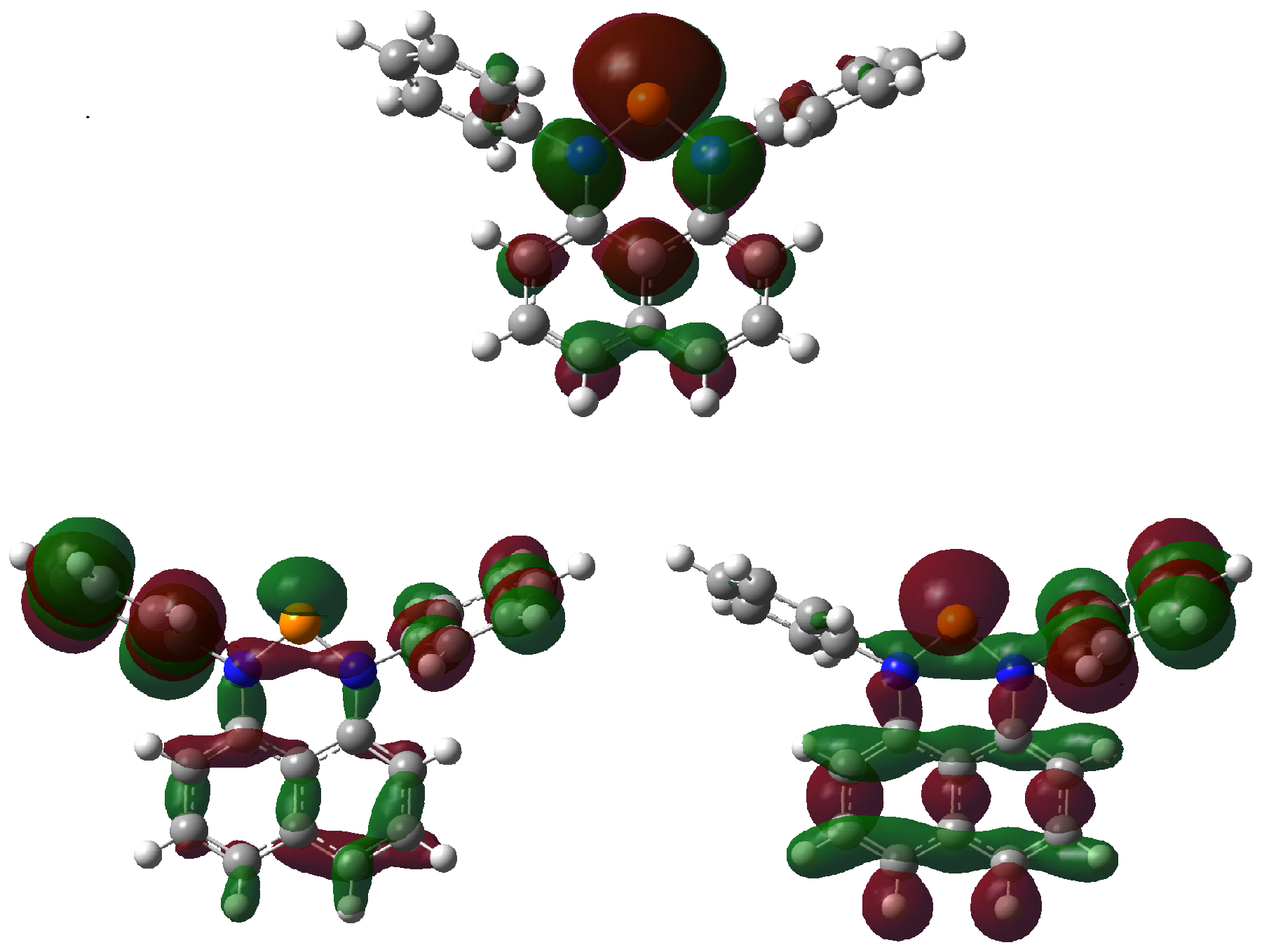

Figure S3. Orbital contour plots of $\left[\mathrm{P}(\mathrm{PhN})_{2} \mathrm{C}_{10} \mathrm{H}_{6}\right] \mathrm{GaCl}_{4} \mathbf{1 0 b}$ showing the LUMO (top) and $\mathrm{P}-$ centered lone pair HOMO-9 (lower left) and HOMO-10 (lower right) obtained from DFT calculations. The HOMO-9 and HOMO-10 display some mixing with the Ph rings and are nearly degenerate. 
The HOMO-LUMO transition for the $\pi$-stacked phosphenium complex 10a was determined to be an excitation is from a naphthalene-centric HOMO to a N-P-N centric LUMO shown in the following Figure S4. The B3LYP/6-311G* spectra predict a blue/violet colour for this species. Computations on compounds 10b-d lead to similar predicted transitions as summarized in Table S1.

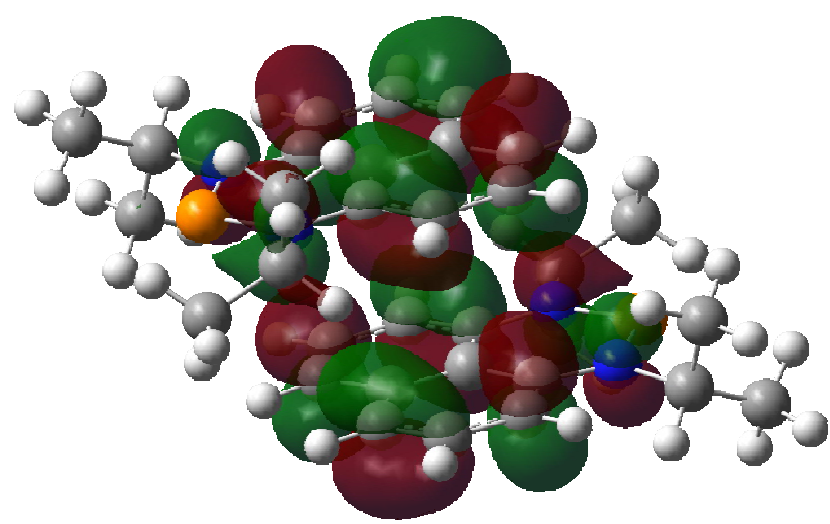

HOMO

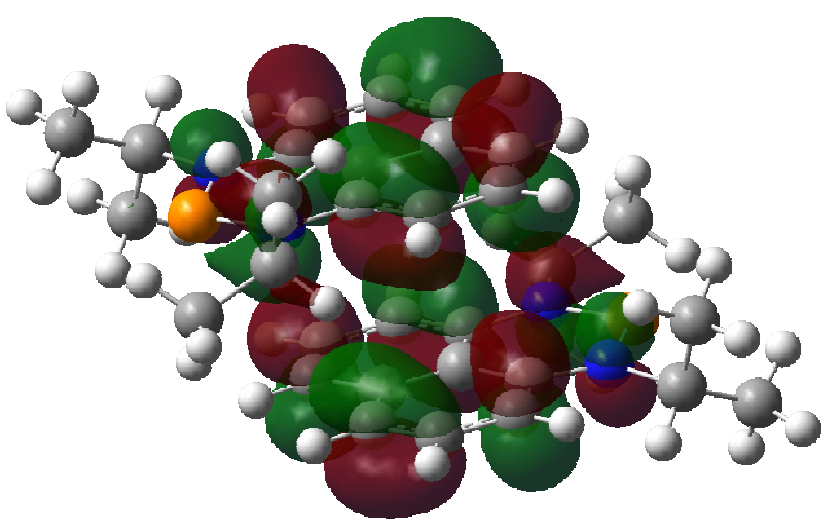

LUMO

Figure S4. HOMO and LUMO orbitals for the stacked ${ }^{i} \mathrm{Pr}-$ phosphenium cations 10a. 
Table S1. The B3LYP/6-311G* calculated electronic transition spectra on compounds 10a-d.

\begin{tabular}{|c|c|c|}
\hline Compound & Absorbances $^{1}$ & Predicted Color \\
\hline $10 a$ & $\begin{array}{l}515.06 \mathrm{~nm} \mathrm{f}=0.0087 \\
324.73 \mathrm{~nm} \mathrm{f}=0.0676 \\
310.69 \mathrm{~nm} \quad \mathrm{f}=0.0398 \\
296.12 \mathrm{~nm} \quad \mathrm{f}=0.0429 \\
293.34 \mathrm{~nm} \quad \mathrm{f}=0.0041 \\
286.48 \mathrm{~nm} \quad \mathrm{f}=0.0689 \\
286.25 \mathrm{~nm} \quad \mathrm{f}=0.0452 \\
273.38 \mathrm{~nm} \quad \mathrm{f}=0.0949\end{array}$ & Red \\
\hline $10 \mathrm{~b}$ & $\begin{array}{r}618.61 \mathrm{~nm} \mathrm{f}=0.0125 \\
427.43 \mathrm{~nm} \mathrm{f}=0.0007 \\
418.78 \mathrm{~nm} \mathrm{f}=0.0139 \\
413.07 \mathrm{~nm} \mathrm{f}=0.0059 \\
378.02 \mathrm{~nm} \mathrm{f}=0.0084 \\
377.09 \mathrm{~nm} \mathrm{f}=0.0049 \\
368.48 \mathrm{~nm} \mathrm{f}=0.0259 \\
365.14 \mathrm{~nm} \mathrm{f}=0.0052\end{array}$ & Green \\
\hline 10c & $\begin{array}{l}628.95 \mathrm{~nm} \mathrm{f}=0.0164 \\
441.58 \mathrm{~nm} \mathrm{f}=0.0011 \\
436.48 \mathrm{~nm} \mathrm{f}=0.0001 \\
419.31 \mathrm{~nm} \mathrm{f}=0.0006 \\
389.52 \mathrm{~nm} \mathrm{f}=0.0086 \\
377.38 \mathrm{~nm} \mathrm{f}=0.0053 \\
376.07 \mathrm{~nm} \mathrm{f}=0.0044 \\
368.02 \mathrm{~nm} \mathrm{f}=0.0016 \\
346.75 \mathrm{~nm} \mathrm{f}=0.0006 \\
343.27 \mathrm{~nm} \mathrm{f}=0.1507 \\
325.70 \mathrm{~nm} \mathrm{f}=0.0477 \\
306.55 \mathrm{~nm} \mathrm{f}=0.0418\end{array}$ & Blue \\
\hline 10d & $\begin{array}{l}800.69 \mathrm{~nm} \mathrm{f}=0.0116 \\
455.50 \mathrm{~nm} \mathrm{f}=0.0014 \\
444.66 \mathrm{~nm} \mathrm{f}=0.0168 \\
439.72 \mathrm{~nm} \mathrm{f}=0.0008 \\
413.24 \mathrm{~nm} \mathrm{f}=0.0145 \\
396.92 \mathrm{~nm} \mathrm{f}=0.0130 \\
389.36 \mathrm{~nm} \mathrm{f}=0.0204 \\
386.41 \mathrm{~nm} \mathrm{f}=0.0011 \\
385.83 \mathrm{~nm} \mathrm{f}=0.0061 \\
375.41 \mathrm{~nm} \mathrm{f}=0.0076 \\
354.21 \mathrm{~nm} \mathrm{f}=0.0133 \\
351.07 \mathrm{~nm} \mathrm{f}=0.0077\end{array}$ & Orange-Yellow \\
\hline
\end{tabular}

\footnotetext{
${ }^{1} \mathrm{f}$ respresents the oscillator strength of the transition
} 
Full citation for reference 19:

Gaussian 03, Revision D.01, M. J. Frisch, G. W. Trucks, H. B. Schlegel, G. E. Scuseria, M. A. Robb, J. R. Cheeseman, J. A. Montgomery, Jr., T. Vreven, K. N. Kudin, J. C. Burant, J. M. Millam, S. S. Iyengar, J. Tomasi, V. Barone, B. Mennucci, M. Cossi, G. Scalmani, N. Rega, G. A. Petersson, H. Nakatsuji, M. Hada, M. Ehara, K. Toyota, R. Fukuda, J. Hasegawa, M. Ishida, T. Nakajima, Y. Honda, O. Kitao, H. Nakai, M. Klene, X. Li, J. E. Knox, H. P. Hratchian, J. B. Cross, C. Adamo, J. Jaramillo, R. Gomperts, R. E. Stratmann, O. Yazyev, A. J. Austin, R. Cammi, C. Pomelli, J. W. Ochterski, P. Y. Ayala, K. Morokuma, G. A. Voth, P. Salvador, J. J. Dannenberg, V. G. Zakrzewski, S. Dapprich, A. D. Daniels, M. C. Strain, O. Farkas, D. K. Malick, A. D. Rabuck, K. Raghavachari, J. B. Foresman, J. V. Ortiz, Q. Cui, A. G. Baboul, S. Clifford, J. Cioslowski, B. B. Stefanov, G. Liu, A. Liashenko, P. Piskorz, I. Komaromi, R. L. Martin, D. J. Fox, T. Keith, M. A. Al-Laham, C. Y. Peng, A. Nanayakkara, M. Challacombe, P. M. W. Gill, B. Johnson, W. Chen, M. W. Wong, C. Gonzalez, and J. A. Pople, Gaussian, Inc., Wallingford CT, 2004. 


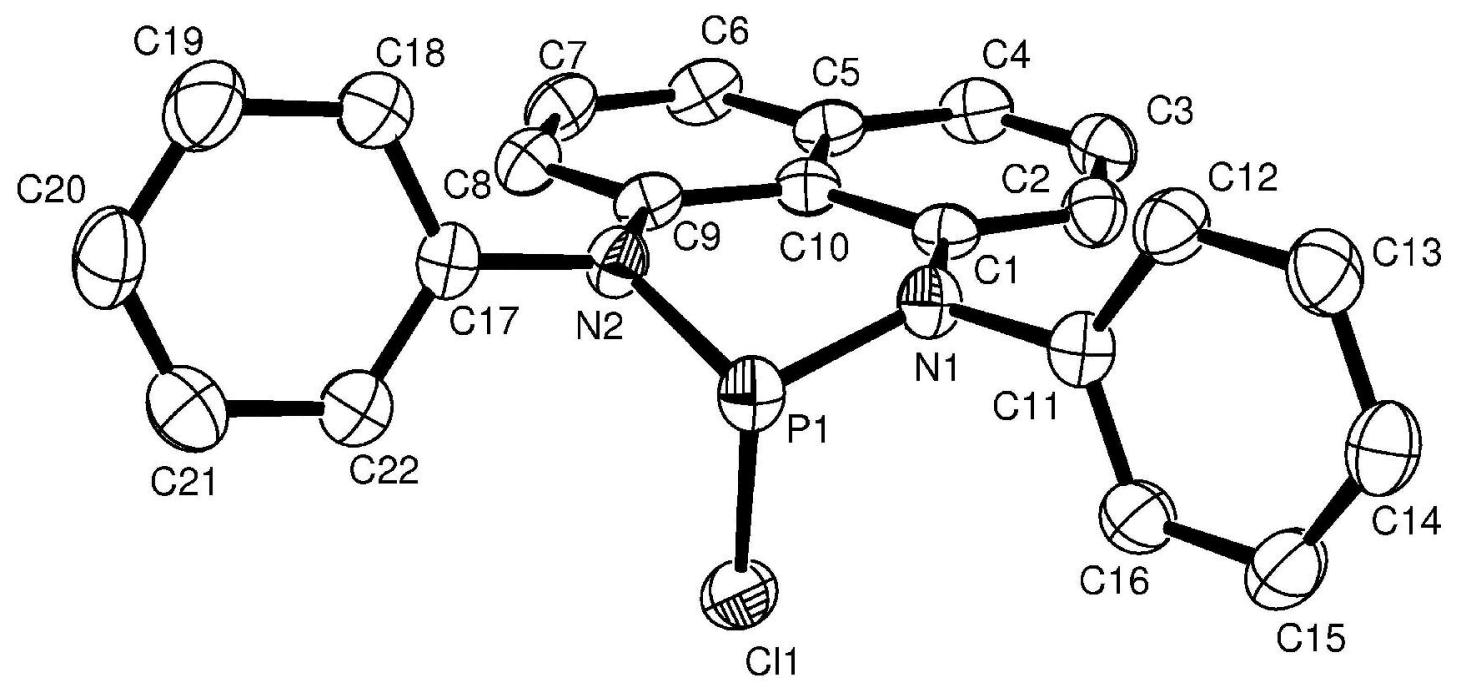

Figure S5. Thermal ellipsoid plot showing the molecular structure and atom numbering scheme for compound 9b. Hydrogen atoms have been omitted for clarity. 

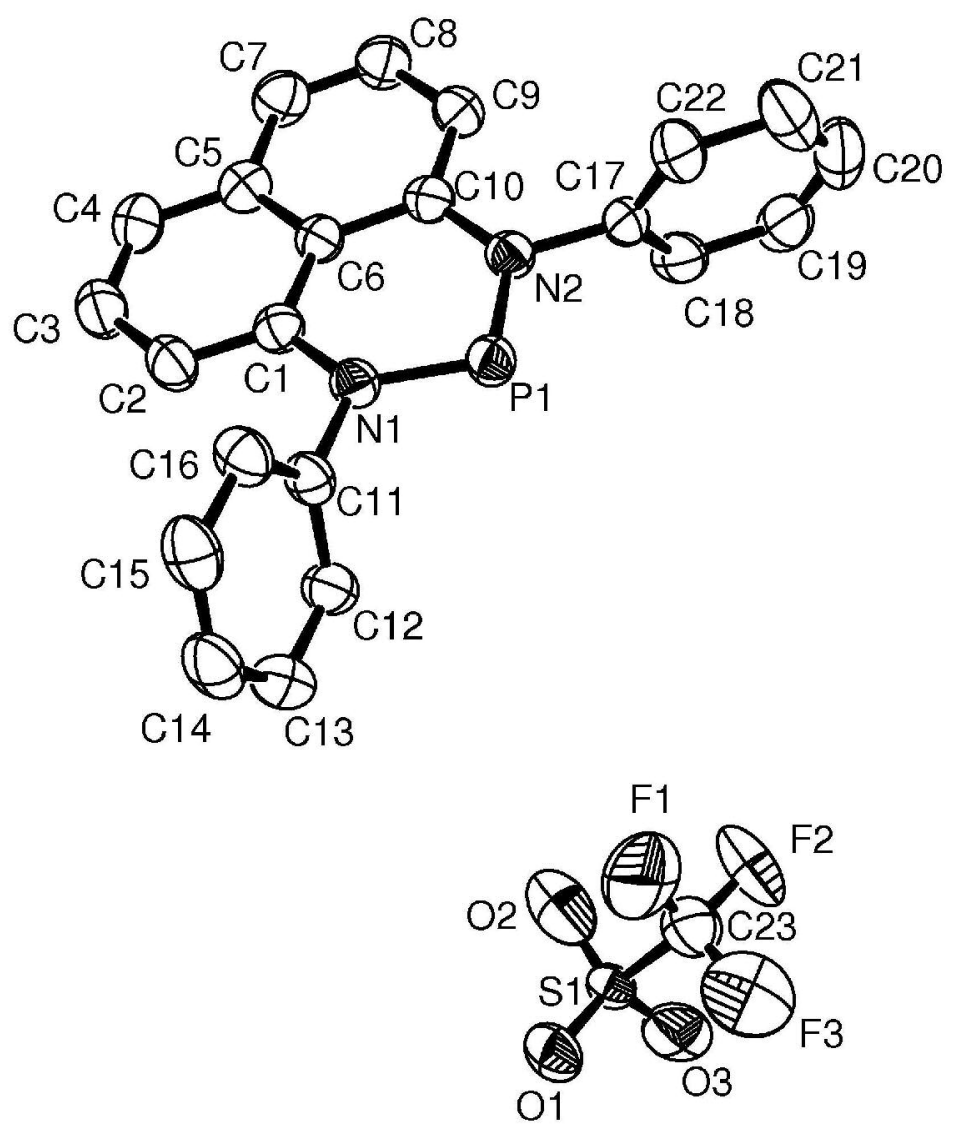

Figure S6. Thermal ellipsoid plot showing the molecular structure and atom numbering scheme for compound 11b. Hydrogen atoms have been omitted for clarity.

NMR data for N,N'-diphenyl-1,8-diaminonaphthalene

${ }^{1} \mathrm{H}$ NMR $\left(\mathrm{C}_{6} \mathrm{D}_{6}\right): 6.9(\mathrm{~m}, 6 \mathrm{H}, H \mathrm{Ar}), 7.2\left(\mathrm{q},{ }^{3} \mathrm{~J}_{\mathrm{HH}}=8 \mathrm{~Hz}, 6 \mathrm{H}, H \mathrm{Ar}\right), 7.3\left(\mathrm{t},{ }^{3} \mathrm{~J}_{\mathrm{HH}}=8 \mathrm{~Hz}, 2 \mathrm{H}, H A r\right)$, $7.5\left(\mathrm{~d},{ }^{3} \mathrm{~J}_{\mathrm{HH}}=8 \mathrm{~Hz}, 2 \mathrm{H}, H \mathrm{Ar}\right)$. The N-H protons were not observed. ${ }^{13} \mathrm{C}\left\{{ }^{1} \mathrm{H}\right\} \mathrm{NMR}\left(\mathrm{CDCl}_{3}\right): 117$ (s, CHAr), 118 (s, CHAr), 121.2 (s, CHAr), 121.4 (s, CAr), 123 (s, CHAr), 126 (CHAr), 129 (s, CHAr), 137 (s, CAr), 140 (s, CAr), 145 (s, CAr). 
